\title{
LA ORIGINALIDAD \\ DE LA ECONOMÍA MARGINALISTA DE JEREMY BENTHAM
}

\author{
ESTRELLA TRINCADO AZNAR*
}

Resumen: En este artículo, se demuestra que la teoría de Jeremy Bentham es precursora de la economía marginalista. El autor introdujo las ideas de utilidad marginal decreciente, la equimarginalidad en el intercambio, realizó una clasificación entre bienes superiores e inferiores y su definición de la propiedad como una expectativa de posesión le llevó a desarrollar una teoría económica basada en la búsqueda individual de información. Así mismo, dio especial importancia a la figura del empresario innovador y describió el mercado como un proceso de descubrimiento en que el agente central es el empresario.

Palabras clave: Bentham, economía marginalista, empresario.

Abstract: In this paper, we present some Bentham's theory features that make him precursor of marginalist economics. The author described the ideas of decreasing marginal utility and equimarginality, he made a commodity classification that distinguished between an upper and a lower class of goods depending on the proximity to use and his definition of property as an expectation of possession lead him to develop an economic theory based on individual information search. At the same time, he attached special importance to the figure of the innovative entrepreneur and described the market as a discovery process in which the central agent is the entrepreneur.

Key words: Bentham, marginalist economics, entrepreneur.

Clasificación JEL: B12.

$\left(^{*}\right)$ Universidad Complutense de Madrid. Agradezco los comentarios de Carlos Rodríguez Braun.

Procesos de Mercado: Revista Europea de Economía Política Vol. II, n. ${ }^{\circ}$ 2, Otoño 2005, pp. 119 a 149 


\section{INTRODUCCIÓN}

En este artículo demostraremos que la obra del inglés Jeremy Bentham (1748-1832) fue precursora de la economía marginalista y que vislumbró algunas ideas austriacas. Innovador en un tiempo en que la teoría del valor ricardiana era el mainstream en las universidades inglesas, introdujo, como es sabido, las ideas de utilidad marginal decreciente y algo parecido a la equimarginalidad en el intercambio. Sin embargo, también llevó a cabo una clasificación entre bienes superiores e inferiores, y su definición de la propiedad como una expectativa de posesión le llevó a desarrollar una teoría económica basada en la búsqueda individual de información. Asimismo, dio especial importancia a la figura del empresario innovador y describió el mercado como un proceso de descubrimiento de las utilidades en que el agente central es, justamente, el empresario (o projector).

\section{PROPIEDAD Y EXPECTATIVA}

La propiedad, dice Bentham, es únicamente obra de la ley. No es más que una base de la esperanza de sacar un provecho determinado de la cosa que se posee a consecuencia de las relaciones que se tiene con ella por obra de la ley ${ }^{1}$. Bentham, como Hume, considera la propiedad como una relación externa. La esencia de la propiedad es que de ella se deriva un beneficio o placer de actos que tienen su terminación en una cosa externa dada y su comienzo en una persona. La combinación del foco externo y la relación placentera hace a la propiedad fuente de felicidad. Los objetos de propiedad pueden ser reales o corporales y ficticios o incorpóreos, pero siempre se dan sobre un objeto externo.

\footnotetext{
1 Bentham (1981: 118).
} 
Si se admite que es útil para el bien social que el producto del trabajo sea propiedad del trabajador porque refuerza el sentimiento de expectativa sin el cual no trabajaría, debe amenazarse al ladrón con un dolor de igual intensidad al placer de robar. El mantenimiento de la propiedad permite el crecimiento, que revierte, en última instancia, sobre toda la sociedad. Además, Bentham introduce el principio de prevención de la decepción, que será la base de las ramas civiles y penales de la ley de la propiedad. Para Bentham, sin la idea de expectativa, no habría diferencia entre una persona que posee una cosa y un usurpador, si ambos sienten igual placer de poseerla. Hemos de calcular el dolor de la decepción sufrida por alguien que pierde una posesión de un derecho adquirido. Por el principio de nodecepción, el rico espera su opulencia tanto como el pobre sabe de su suerte. Así, y al enfatizar la idea de expectativa, en la transformación de la ley Bentham se basa en un concepto del tiempo futurista, gradualista y continuista.

Es decir, Bentham identifica la propiedad con los sentimientos humanos de placer, seguridad y expectativa, y, especialmente tras la Revolución Francesa, la idea de un cambio revolucionario en la distribución de propiedad le aterroriza.

¡Una revolución en la propiedad! Es una idea que veo con horror... Implica disturbios en las posesiones, desilusión en las expectativas... La opulencia reducida a mendicidad, los frutos de la industria hechos presa de la rapacidad y disipación, la igualación de toda distinción, la confusión de todo orden y la destrucción de toda seguridad. ${ }^{2}$

Por tanto, Bentham acaba rechazando todo cambio social en favor de la racionalización del status quo.

En todos estos casos, ¿cuál debe ser el objeto primario del gobierno? Estabilidad. Éste debe ser el primer objeto de sus deseos en todo punto, si no de sus medidas.

\footnotetext{
2 Stark (1952a: 318), extractado de Supply without Burden.
} 
¿Por qué? Lo que se pierde para un hombre por un cambio del precio es ganancia para otro, siendo ambos miembros de la comunidad y el interés de cada uno una porción igual del interés de la comunidad. Cierto: pero la constitución de la naturaleza humana es tal que el disfrute resultante de una ganancia nunca es igual al sufrimiento resultante de una pérdida.

La estabilidad de los precios es una rama de esa especie de seguridad que tiene a la propiedad como objeto. ${ }^{3}$

En cualquier caso, según Bentham, siguen existiendo razones para buscar la igualdad: el pobre puede valerse del principio de minimización de la desigualdad y, además, es cierto que una masa de riqueza dada produce más felicidad cuando está repartida entre más número de individuos. Pero toda desigualdad que no tenga una utilidad especial para justificarse es una injusticia ${ }^{4}$.

De todos modos, en la teoría de Bentham no hay relación entre el derecho de propiedad y los derechos humanos, ni una crítica de la relación del hombre con otros hombres y con la naturaleza ${ }^{5}$. Estaba en contra de la esclavitud pero no por motivos morales, sino porque la libertad hace a los trabajadores más productivos. Sin embargo, como la experiencia no ha enseñado a los esclavos otra condición, éstos no perciben su sujeción como abominable y no se verían decepcionados por seguir en esa condición ${ }^{6}$. Diferenciaba entre la trata de esclavos, que desaprobaba (los españoles e ingleses, de hecho, negociaron su prohibición ya en 1817) y la esclavitud misma, que se resistió a condenar taxativamente. De hecho, parecía más preocupado por la suerte de los animales que por la de los esclavos ${ }^{7}$.

3 Stark (1952a: 364-5), extractado de Supply without Burden.

4 Stark (1952a: 329), extractado de Supply without Burden.

5 Mises (2001: 209) también criticó la teoría del Derecho Natural, explícitamente basándose en Bentham. Sin embargo, en esto no coincide con otros Austriacos. Por ejemplo, Murray N. Rothbard (1995) ha adoptado una postura iusnaturalista.

6 Bentham (1981: 196).

7 Rodríguez Braun (1989: 126). Campos Boralevi ha estudiado la postura de Bentham sobre la esclavitud y los animales en Campos Boralevi (1984: 142-175). 


\section{VALOR-UTILIDAD: EL CÁLCULO FELICÍFICO}

Adam Smith ha echado luz sobre mucho humo; pero hay casos también donde ha echado humo donde antes había luz. ${ }^{8}$

Ésta es la descripción que Bentham hace en The True Alarm de la teoría del valor Smithiana, y en términos parecidos criticará, en el margen de Table of the Springs of Action, la idea de la propensión a intercambiar (¿por qué no decir que los individuos maximizan su utilidad? $)^{9}$.

En The True Alarm, Bentham define la riqueza en base a dos elementos: debe haber un objeto sensible y el objeto debe ser materia de uso y deseo, tener atractivo para los hombres. En el capítulo 5 discute la noción de valor: Todo valor se funda en la utilidad.

En la antología de escritos económicos de Bentham que hace Stark, se incluye una relación de extractos que el editor llama «la psicología del hombre económico» (unas ideas que luego serían usadas por la escuela de utilidad marginal). La felicidad para Bentham es una «idea», la expectativa del futuro placer y dolor que influye en la acción interesada y esperada. El hombre, según Bentham, busca maximizar una suma de placeres y minimizar una suma de dolores esperados. Pero ¿hay acciones impulsivas precedentes a ese cálculo, con magnitudes positivas-negativas? Incluso la pasión calcula, dice Bentham, aunque de modo confuso, y, además, para Bentham, la pasión no es un modo de acción habitual. La proposición de que la pasión no calcula, no es verdad, como casi todas las proposiciones generales y oraculares... Todos los hombres calculan ${ }^{10}$.

\footnotetext{
8 Stark (1952c: 80), extractado de The True Alarm.

9 Bonner (1995).

10 Stark (1952c: 434), extractado de The Psychology of Economic Man: XXIII. En esto coincide con algunos austriacos. Popper sintetiza una idea central de la economía austriaca: «Las instituciones sociales no proyectadas nacen como conse-
} 
Bentham apunta que la cuantificación de experiencias físicas puede perfeccionarse y producir números concretos sobre valoraciones mentales. Pero esas valoraciones se producen, de facto, siempre que se menciona un precio monetario. El dinero mide experiencias físicas que sólo el sufridor puede valorar. Es decir, el dinero puede ser medida de placer o dolor. Sin embargo, ésta está sujeta a una «objetividad subjetiva». De hecho, el valor de la unidad monetaria es alto para los que tienen poca propiedad y bajo para los que tienen mucha. Por tanto, el valor del dinero relativo sí es objetivo, y podemos hacer comparaciones interpersonales de utilidad entre los hombres a los que podría idealmente medirse su umbral de placer. Si ceteris paribus dos hombres en iguales circunstancias pierden la misma cantidad de dinero, podemos suponer que pierden la misma cantidad de felicidad.

Esto es así para los bienes de valor esencial e invariable, como las necesidades relativas. Sin embargo, hay bienes de valor variable y caprichoso, como los lujos relativos, cuya comparación de utilidad interpersonal es más difícil. Bentham diferencia, como Smith, entre valor en uso y valor en cambio, aunque, según Bentham, a propósito de esta distinción Adam Smith es criticable: el agua puede tener valor de cambio en algunas situaciones, mientras que los diamantes pueden tener sus usos ${ }^{11}$. Pero, con más originalidad, Bentham diferencia entre valor intrínseco y valor convencional. El valor intrínseco es la base del valor convencional, igual que el valor en uso es la base del valor en cambio.

cuencias involuntarias de acciones racionales» [Popper (1961: 88)]. Mises, de hecho, manifestaba que «todo orden social fue pensado y proyectado antes de ser puesto en práctica» [Mises (2001: 225)]. Sin embargo, como dice Huerta de Soto [en la introducción de Mises (2001: 1xi)], hay tres tendencias en la Escuela Austriaca: la racional-utilitarista de Mises, el evolucionismo de Hayek y el iusnaturalismo de Rothbard y Hoppe.

11 Stark (1952c: 19), extractado de Introduction. 
Nótese que el valor en el sentido de cambio surge de, y depende de, y está proporcionado con, su valor en el sentido de uso: porque ningún hombre dará nada que tenga valor en el sentido de uso en cambio de nada que no tenga ese valor.

Pero el valor en el sentido de uso puede diferenciarse en el general, o el que tiene lugar en tanto, y no más lejos que, la cosa es útil para las personas en general; y en el especial o idiosincrásico, que tiene lugar en tanto que en el caso de esta u otra persona en particular la cosa tiene un valor en el sentido de uso además del valor que tiene en el caso de las personas en general: un ejemplo del cual es el «pretium affectionis», el valor de la afección. ${ }^{12}$

Hemos de decir que James Mill se hizo seguidor del benthamismo en política y gobierno, pero no intentó acercarse al análisis de placer-dolor de Bentham, sino que se basó en la aproximación física de Ricardo a los problemas de producción y distribución ${ }^{13}$. Y a través de Mill padre e hijo y de Mc Culloch, son las ideas ricardianas del valor las que dominan la economía política inglesa hasta $1860^{14}$. Bentham, de hecho, fue crítico de la economía de Ricardo. En los libros de la Renta de Ricardo, no hay mucha lógica. Quise que los corrigiera en estos particulares, pero no era consciente de ello, y Mill no lo deseaba. Confundía el valor

12 Stark (1952a: 108-9), extractado de The Philosophy of Economic Science: XXV.

13 Sus Elementos de Economía Política eran un tratado didáctico que incorporaba las doctrinas de Ricardo. En él, escribe que el valor relativo de los bienes depende de la oferta y demanda, en primer lugar, pero del coste de producción en último término. Incluso reduce la teoría del valor a cantidad de trabajo. No expone la idea de que la utilidad es una razón necesaria, aunque no suficiente, para que los bienes posean valor.

14 Según Halévy, sólo puede considerarse benthamita en la primera parte del siglo XIX a Samuel Bailey, que en 1825 publica A Dissertation on the Nature, Measures and Causes of Value, una de las más penetrantes críticas a Ricardo del XIX. Le llamaban «the Bentham of Hallamshire». Creía que las leyes de economía política son expresa operación de ciertos motivos de la mente humana, visión que contrasta con la teoría física y material de Ricardo. Éste fue criticado por James Mill en la Westminster Review, en enero de 1826, en un artículo anónimo. Se ve la dicotomía entre la política benthamita y la economía ricardiana, con la que se identifican los clásicos [Halévy (1928: 353-4)]. 
con el coste ${ }^{15}$. Sus ideas no fueron muy tenidas en cuenta, excepto las de Defence of Usury. Además, lo que Bentham escribe sobre valor, producción y distribución, crecimiento, empleo o dinero, no fue accesible a los economistas del siglo XIX, o sólo tras el año 1843, a través de la edición de Bowring ${ }^{16}$.

\section{BIENES DE ORDEN SUPERIOR E INFERIOR}

Sin embargo, la teoría de Bentham está cercana a la posterior metodología mengeriana, no sólo por el subjetivismo, sino porque también diferencia entre bienes de orden superior e inferior, de producción o de consumo, y se basa en la cercanía al uso como parámetro diferenciador ${ }^{17}$. Según Bentham, hay bienes de valor inmediato o intrínseco, como la comida; y bienes de valor derivado o servil, como las herramientas.

La utilidad inmediata no admite de grados; pero la utilidad no inmediata puede situarse en grados en el número que se quiera. La «escala» a la que estos grados pertenecen puede llamarse la escala de «vecindad al uso». Los instrumentos cuya estación está en el mayor grado en la escala — digamos el primer grado, el grado más cercano al uso inmediato- pueden llamarse instrumentos de primer orden: aquellos más cercanos a ellos, e.d. más cercanos bajo ellos, instrumentos de segundo orden, y así siguiendo, a través de cualquier número de grados

15 Bentham Works X, 498; citado en Stark (1952c: 48), extractado de Introduction.

16 Collison Black (1988). Sin embargo, Jevons, en 1871, en su Theory of Political Economy, vuelve al principio de la mayor felicidad y a la consideración del placer y dolor como motivo de la acción. Cita a Bentham en varias ocasiones y cuando procede a la cuestión del "placer y dolor como cantidades», en el capítulo II, llega a medir placeres y dolores por su intensidad, duración, certidumbre, cercanía, fecundidad, pureza y extensión, como hacía Bentham.

17 Sin embargo, aunque analizaban procesos similares, partían de concepciones pre-analíticas de diversa índole. Véase Menger (1984). 
que, en cualquier sistema de instrumentos conectados, pueden en cualquier momento encontrarse ejemplificados. ${ }^{18}$

En la distinción entre la parte primaria y la derivada de la renta nacional, y entre el ingreso natural y convencional, hay un eco en Bentham de la doctrina fisiocrática. La diferencia entre agricultura, manufactura y artesanía, dice Bentham, es la mayor división del trabajo, la mayor cantidad de usos intermedios empleados conjuntamente y el número de grados en los que los instrumentos se acercan en la escala de «vecindad al uso», es decir, el incremento del tiempo de producción.

Es característico de la teoría de Bentham el hecho de que incluya a las personas, como fuente de servicios, entre la riqueza. Los habitantes son parte del capital productivo del país. Además, el trabajo es «sólo» un bien instrumental, no uno de orden inferior, que sólo puede compensarse con el placer monetario del salario.

Aversión —no deseo- es la emoción, la única emoción que el trabajo, tomado por sí mismo, puede producir... En tanto que el trabajo se tome en su sentido propio, el amor al trabajo es una contradicción en los términos. ${ }^{19}$

18 Stark (1952c: 448-9), extractado de The Psychology of Economic Man: XXXIV. Véase Stark (1952c: 84), extractado de The True Alarm; o el siguiente párrafo: «Con respecto a "la cercanía al uso", la estación de los materiales, que sirven como objetos de la industria, es susceptible de unos grados indefinidamente numerosos... Cuando, considerado bajo toda modificación de la que es susceptible, el trabajo ha sido llevado a un estado que se puede llamar con propiedad "trabajo acabado", si tomamos cualquier artículo de él como ejemplo, se encontrará que es o bien de tal naturaleza que permite, sin intervención de ningún otro objeto, ser aplicado en un modo inmediato al "uso inmediato" —en el sentido de que excluye del dolor o de que administra placer directamente- o que es susceptible de ser aplicado al uso como uso preparatorio, intermedio o digamos instrumental» [Stark (1952c: 448), extractado de The Psychology of Economic Man: XXXIV].

19 Stark (1952c: 428), extractado de The Psychology of Economic Man: IX. 


\section{UTILIDAD MARGINAL DECRECIENTE, INTERCAMBIO Y EQUIMARGINALIDAD}

Las ideas de Bentham están continuamente rozando la de la importancia del principio marginal ${ }^{20}$. En Axioms, el lector encuentra una exposición clara de la idea de «mínimo sensible» de riqueza, una primera aproximación a la utilidad marginal.

Entre el servicio real y su retribución, la medida exacta es la menor cantidad de materia de retribución que aquel que es capaz de prestar el servicio consiente tomar a cambio de él. Ésta es la medida de todos los precios, ésta es la medida del valor de todas las cosas buenas que son valorables y tangibles. ${ }^{21}$

Pero, además, formula también la idea de utilidad marginal decreciente, casi con las mismas palabras que sus continuadores subjetivistas.

La magnitud del placer producido no incrementa en un ratio tan grande como la causa que lo produce. Tómese, por ejemplo, la misma causa que antes: por ejemplo, el dinero. Tómese un individuo: désele una cierta cantidad de dinero, producirás en su mente una cierta cantidad de placer. Désele otra vez la misma cantidad, harás una adición a la cantidad de su placer. Pero la magnitud del placer producido por la segunda cantidad no será dos veces la magnitud del placer producido por la primera. ${ }^{22}$

El principio de utilidad marginal decreciente se aplica no sólo al dinero, sino a toda gratificación: altas dosis de la materia excitante aplicada al órgano hacen que su sensiblidad de algún modo se gaste.

20 Schwartz presentó algunas de estas «Sugerencias marginalistas en Bentham» en Schwartz (1976).

21 Stark (1952a: 118), extractado de The Philosophy of Economic Science: XXVII.

22 Stark (1952c: 441), extractado de The Psychology of Economic Man: XXIX. 
Respecto al intercambio, es posible que Bentham no viera clara la idea de que, dado que los hombres tienen distintas utilidades o recursos iniciales, ambos negociantes puede beneficiarse de voluntariamente sacrificar una porción de su felicidad por su ganancia ${ }^{23}$. Sin embargo, plantea la idea de «intercambio por reparación» e incluso la de indiferencia. Relación hecha al individuo en cuestión, un mal es reparable y exactamente reparado cuando, tras haber sufrido el mal y recibido la compensación, sería un asunto de indiferencia recibir el mismo mal, junto a la misma compensación $n^{24}$.

Sin embargo, Bentham sí habla de la utilidad ganada por la transferencia de bienes. En el Tratado ${ }^{25}$, estudia la patología, las sensaciones, afectos y pasiones y los efectos sobre la felicidad. Sea la modificación del volumen de prosperidad lo que sea, al perder sin obtener un equivalente, un hombre sufre en proporción al valor que estima de él - el valor que les da... ${ }^{26}$ Para juzgar el efecto de una porción de riqueza sobre la felicidad la considera en tres estados diferentes: 1) Cuando siempre ha estado en las manos de los interesados. 2) Cuando acaba de salir de ellas. 3) Cuando acaba de entrar en ellas.

I. Caso primero. Las cantidades de riqueza en cuestión están en las manos de las dos partes en cuestión: ni entrando ni saliendo de las manos de ninguno.

1. Ceteris Paribus: a cada partícula de volumen de riqueza corresponde una partícula del volumen de felicidad...

5. Mínimo de riqueza, digamos 10 libras por año; sea el mayor exceso de felicidad producido por un exceso en la cantidad de riqueza de 2 a 1; magnitud de la partícula de riqueza, 1 libra por año. Sobre estos datos puede basarse una escala o cuadro, que exhibiría las cantidades de felicidad producida por

\footnotetext{
23 Schwartz (1986: 74-103).

24 Stark (1952c: 438), extractado de The Psychology of Economic Man: XXVI.

25 Bentham (1981: 110-6).

26 Stark (1952a: 108), extractado de The Philosophy of Economic Science: XXV.
} 
cada una de las adiciones hechas a la cantidad de riqueza en el fondo de la escala, dado que hay muchas libras entre 10 y $10.000 \ldots$

II. Caso en que las partículas de riqueza estén a punto de pasar a las manos de las partes.

1. Fortunas desiguales: una partícula de riqueza añadida al que tiene menos produce más felicidad que al que tiene más...

3. En estos datos puede basarse una escala que exhiba cantidades de felicidad producidas por adiciones al mínimo de riqueza a la felicidad respectiva de cualquier número de personas, cuyas respectivas cantidades de riqueza exceden una a otra por la cantidad de una partícula.

III. Caso tercero, las partículas de riqueza están a punto de salir de las manos de las partes...

6. Cuanto mayor es la fortuna del individuo en cuestión, mayor es la probabilidad de que, por la sustracción de una cantidad dada de su volumen de riqueza, no se haga ninguna sustracción de la cantidad de su felicidad. ${ }^{27}$

Bentham, con su método geométrico, que muestra las consecuencias estáticas sobre el placer de una transferencia, estaba pensando en una escala que nos recuerda los gráficos de utilidad que posteriormente realizarían los economistas. Además, casi llega a formular el principio de equimarginalidad en el intercambio, aunque no logra definirlo con nitidez.

IV. Supongamos las partículas de riqueza a punto de irse de las manos de una parte a la de otros.

1. Fortunas iguales: tómese de una parte una porción del volumen de riqueza y entréguese al otro, la cantidad de felicidad obtenida por el que ha incrementado su riqueza no será tan grande como la cantidad de felicidad perdida por el que la ha perdido.

2. Fortunas desiguales: cuanto más pobre sea el perdedor, más rico será el ganador; mayor es la disminución producida en la masa de felicidad por la transferencia que en el último caso mencionado. ${ }^{28}$

27 Stark (1952a: 113-4), extractado de The Philosophy of economic Science: XXV.

28 Stark (1952a: 114-5), extractado de The Philosophy of economic Science: XXV. 


\section{LA INFORMACIÓN}

Tratando el tema del precio y del coste, Bentham dice que para estimar el poder de compra sólo deben tomarse en cuenta los «precios definitivos» - pagados por el consumidor, de los bienes de primer orden- no los «precios preliminares» - de los bienes de segundo orden-. Para Bentham sólo se incrementa el stock de riqueza con ayuda del capital, el resultado del trabajo precedente $^{29}$. Pero, a pesar del parecido de esta afirmación con la teoría de Ricardo, en Institute of Political Economy Bentham muestra una teoría de los factores de producción diferente de la de Ricardo. Distingue tres factores: inclinación, conocimiento y poder (la voluntad de producir riqueza; el conocimiento de cómo producirla; y el poder sobre las cosas externas necesario para ese propósito). La inclinación se da en cantidad ilimitada, fluye de la constitución del hombre y no requiere ser incentivada. El conocimiento es fruto de la inclinación, con lo que el legislador tampoco tiene que incentivarlo. El poder, es decir, el control sobre el capital y bienes de capital, es el factor de producción limitante, dado que es inevitablemente escaso. Pero es poco posible que el gobierno tome pasos efectivos para evitar esa escasez de un factor natural. Por tanto, en conjunto el laissezfaire es la actitud apropiada.

La ventaja de la dirección del capital empleada en la comunidad depende de: 1 . la elección del comercio y 2. la elección del modo de llevarlo a cabo. La posibilidad de mejor elección de la dirección del capital, según Bentham, se dará en proporción al grado de interés que el que elige pone en hacer la elección; y en proporción al mayor conocimiento y juicio del nego$\mathrm{cio}^{30}$. La posibilidad de que un hombre posea en un grado

29 Stark (1952a: 196), extractado de Defence of Usury. Proscript. Short Observations on the Injustice and Impolicy of Forced Reductions of the rate Of Interest.

30 Stark (1952a: 228), extractado de Manual of Political Economy. 
superior las facultades de conocimiento y juicio depende en gran medida del grado de interés que pone en poseerlas. El interés que un hombre toma en los asuntos de otros, por ejemplo un miembro de la soberanía en el de los súbditos, no es probable que sea tan grande como el interés que cada uno toma en sí mismo; mucho menos cuando ese otro es un perfecto extraño para él ${ }^{31}$.

Según Bentham, los bienes que se pueden propagar con externalidades positivas son los que el Estado más debe fomentar. Ha sido celebrada la diferencia que hace, tanto en Manual como en Institute, entre acciones sponte acta, agenda y non agenda. Según Bentham, no hay una relación correcta de estas tres clases de acciones. La regla general de la intervención consiste en que para estimar la deseabilidad de cualquier gasto del gobierno en un subsidio debe compararse su uso probable con la carga del impuesto más nocivo en vigor, por la simple razón de que ese impuesto podría ser abolido si no fuera por el incentivo concedido de ese modo (vemos que Bentham utiliza la idea de marginalidad en este caso) ${ }^{32}$.

Para que el Estado logre un incremento de la riqueza, por ejemplo, debe crear una inclinación e incentivos, transfiriendo riqueza de una rama a otra. Pero, dice Bentham, todo lo que incentiva A desincentiva a $B$, a no ser que la cantidad de capital fuera ilimitada o que el agente público tuviera información sobre el interés privado o algún interés en la rama de comercio.

Es falso porque las dos proposiciones son falsas; pero si ambas fueran verdaderas, la práctica podría no ser errónea. Si fuera más probable que el hombre de Estado buscara en este

31 Stark (1952a: 229), extractado de Manual of Political Economy.

32 «Regla práctica que se supone nueva: para juzgar la conveniencia y elegibilidad de una rama nueva de gasto, comparar su beneficio con la carga de la porción correspondiente del producto del impuesto más gravoso» [Stark (1952c: 367), extractado de Institute of Political Economy]. 
particular el verdadero interés del individuo más que el mismo individuo, la necesaria limitación de la cantidad de capital de la nación no sería suficiente argumento contra su interferencia. ${ }^{33}$

Efectivamente, según Bentham, el problema del gobierno es la información asimétrica. Pero sí éste tuviera más capacidad de obtener y difundir información para que los individuos se inclinen a la rama más beneficiosa de industria, no puede haber daño en su difusión a costa del gobierno, dado que incluso por el mero avance de una pequeña porción de dinero bien aplicado, puede difundirse una infinidad de conocimiento útil ${ }^{34}$.

Pero el gobierno tiene muy difícil resolver su problema de agencia, y sólo es mejor que el individuo en los casos en que puede beneficiar su superioridad en lo que respecta a la longevidad: para el gobierno, el principal es un ser ideal; sin embargo, el manager, que conoce directamente a su agente, no tiene tantos problemas de agencia.

Los individuos actúan para sí mismos: los agentes del gobierno actúan para otros... El principal del agente privado es una persona a la que conoce, una persona a la que ve, una persona que le vigila o que está siempre preparado para vigilarle, una persona a la que está obligado, una persona por la que siente naturalmente un afecto, a la que normalmente ha tenido un afecto y ha tenido una buena opinión de él. El principal del agente del gobierno es un ser ideal, al que nadie conoce y del que nadie se preocupa. ${ }^{35}$

Por otra parte, para Bentham es fundamental el proceso de descubrimiento de la información o el conocimiento. El subjetivismo de la información dispersa y la incertidumbre que

\footnotetext{
33 Stark (1952a: 234-5), extractado de Manual of Political Economy.

34 Stark (1952c: 359), extractado de Institute of Political Economy.

35 Stark (1952b: 146-7), extractado de Plan for the Augmentation of Revenue.
} 
implica un proceso de descubrimiento individual e intransferible no nos permite objetivizar la utilidad. El problema de la agencia del gobierno es que éste no sabe lo que quieren los individuos e interviniendo puede obstruir sus objetivos ${ }^{36}$. Es posible que su visión atomística de la sociedad de Bentham no le capacite para asegurar un «orden espontáneo». Los mismos austriacos consideran actualmente que los procesos de descubrimiento actúan esencialmente dentro del desequilibrio dinámico. Sin embargo, asumen que, si las acciones e interacciones de hombres más o menos libres, cuyas redes de información son tejidos de relaciones diarias y de secretos comerciales, no llegan a un orden, menos podemos asegurar que la compulsión de un sistema lo logre. Las instituciones son importantes, en este caso, porque juegan un papel crucial en la producción, transmisión y uso de la información y la información aprendida dentro de las instituciones no estatales hacen la acción humana previsible, y mantienen las expectativas. Y dice Bentham en un pie de página respecto al interés del gobierno de recoger esa información:

Pie. Oportunidad para recoger esa información particular, tiempo necesario para reflexionar sobre ello, interés en formarse un juicio correcto, en todos estos particulares siempre está en desventaja respecto a las personas mismas que desea ver empleadas de esa manera. ${ }^{37}$

36 «No es frecuente que un hombre sea un mejor juez de otro que el otro es para sí mismo, incluso en los casos en que el consejero se toma las molestias de tomar todos los materiales para juzgar, que están al alcance de la persona a que aconseja. Pero el legislador no está en posesión, ni puede estarlo, de ninguno de esos materiales» [Stark (1952a: 140), extractado de Defence of Usury].

37 Stark (1952a: 202), extractado de Defence of Usury. 


\section{LA FIGURA DEL EMPRESARIO}

Por todo lo que hemos comentado, Bentham da especial valor a la figura del empresario. Criticó duramente a Smith por haber presentado un decisor que enfrenta el riesgo como una rutina más. En La Riqueza de las Naciones no está clara la diferencia atribuida a Say entre el premio del empresario como organizador y el premio del capitalista ${ }^{38}$. Bentham, sin embargo, defiende a los proyectistas y especuladores en su Defence of usury, volviendo a la tradición Cantillon-Say ${ }^{39}$. Aunque, como Smith, critica la prodigalidad, reprocha a Smith el haber infraestimado el papel de esos «hombres de talento» que, a través de su invención e imaginación, son responsables del progreso y de la riqueza de las naciones, dado que encuentran nuevos canales de comercio. Aunque las empresas de los proyectistas fallen, la sociedad en conjunto queda intacta porque otros intentarán evitar hacer los mismos errores y las innovaciones introducidas por los proyectistas en el proceso productivo o maquinaria se expandirán a través del sistema económico, cualquiera que sea la suerte de su promotor original. Según Bentham, la innovación es la fuerza que mueve el desarrollo porque lo que es ahora institución, una vez fue innovación ${ }^{40}$.

\footnotetext{
38 Según Schumpeter, Smith no tenía teoría del empresario. Consideraba que el capitalista sólo acumula capital y contrata a los trabajadores productivos, y el negocio va solo. Haciendo esto, el capitalista expone sus medios de producción al riesgo de la pérdida, pero aparte de esta actividad, él sólo supervisa su negocio para asegurar sus beneficios [Schumpeter (1954: 555)]. Sin embargo, Knight dice que Smith reconoce que el beneficio contiene, incluso habitualmente, un elemento que no es el interés del capital [Knight (1947)]. Según Hollander, Smith formuló el concepto de beneficio como un premio a la incertidumbre de la renta ganada con la utilización del capital en el establecimiento de una empresa [Hollander (1973: 170)].

${ }^{39}$ Hébert R.F. y Link A.N. recogen la obra de Bentham sobre el empresario y destacan que sus vínculos con la tradición intelectual francesa eran más fuertes que los de sus contemporáneos [Hébert y Link (1988: 50-54); véase Santos Redondo (1997: 35)].

40 Stark (1952b: 355), extractado de Observations by Sir Frederick Morton Eden (in form of a Letter) on the Annuity Note Plan as contained in the Three first printed sheets with the two tables: with counter-observations by the author of the plan.
} 
Los proyectistas se caracterizan por separarse de los modelos rutinarios de comportamiento, sobresalir de la masa, con lo que son una elite restringida que requiere valor y genio. Cita dos casos en que es necesario sólo el coraje, no el genio: la apertura de un nuevo mercado y la búsqueda de una fuente nueva de oferta ${ }^{41}$. Bentham probablemente quería que Smith revisara sus opiniones sobre la función llevada a cabo por el hombre prudente en su sistema, dado que conocía el papel del hombre prudente en la teoría de Smith y, sin embargo, le recuerda que éste no se embarca en proyectos nuevos ${ }^{42}$.

Bentham dice que el proyectista introduce una mejora ${ }^{43}$, se adentra en senderos desconocidos o bien en la producción de un nuevo bien adaptado al uso humano o en el mejoramiento de su calidad y disminución de su gasto. El término proyectista... se da a todas las personas que, en el cultivo de esas artes que han sido llamadas útiles, dirigen sus esfuerzos a cualquiera de esos departamentos en que su utilidad parece más indudable ${ }^{44}$. Su función, por tanto, es captar la utilidad del bien final anticipadamente, proveyendo los placeres del público en contextos de incertidum-

41 Cuando añadimos los otros dos determinantes de la innovación -la producción de un nuevo bien y la introducción de un nuevo método de producción, tenemos 4 de las 5 combinaciones nuevas que cita Schumpeter en Theory of Economic Development. Bentham anticipó las ideas de Schumpeter sobre el empresario. Es sorprendente que Schumpeter, aparte de una referencia breve [en Schumpeter (1949: 64)] no hace mención a Bentham en el tema de las características y papel central de la función empresarial [Heberton (1949: 337-8)]. Sin embargo, sí consideraba que Bentham había sido quien con más claridad percibió la importancia y naturaleza de la función empresarial, aunque sus opiniones sobre el tema pasaron inadvertidas a los economistas [Schumpeter (1966: 255-272)].

42 Pesciarelli destaca el hecho de que Smith tenga como «hombre ideal» al hombre corriente, moderado y frugal mientras Bentham - y Schumpeter - entronizan al individuo excepcional y aventurero [Pesciarelli (1989: 521-36)]. La visión de Smith contrasta con la de Schumpeter, que dirá que el empresario «camina hacia el éxito sobre el cordel de deudas».

43 Aquí usa cursivas para la palabra «mejora» porque diferencia entre mejora e invención. La mejora es un nuevo método de combinar recursos para propósitos productivos; la invención se da en el progreso científico.

44 Stark (1952a: 170), extractado de Defence of Usury. 
bre $^{45}$. Para Bentham, el empresario es una figura positiva cuando rompe las rutinas empresariales ${ }^{46} \mathrm{y}$, es de suponer, investiga sobre los gustos de los consumidores que, si compran su producto, están saciando un deseo anterior a la compra, aunque sea subconsciente ${ }^{47}$. Pero, ¿cómo es posible, (puede preguntarse), que el motivo por el que un hombre se ve activado sea secreto a sí mismo? Nada es más fácil, nada más frecuente. De hecho, lo raro es, no que no lo conozca, sino que lo conozca ${ }^{48}$. En esto, anticipa la idea Schumpeteriana de innovación como destrucción creadora ${ }^{49}$. El progreso, además, se da por la mejora de los instrumentos ${ }^{50}$.

\section{EL MÁXIMO LEGAL DE TIPO DE INTERÉS}

Defence of Usury surgió de un rumor que había oído Bentham según el cual el ministro Pitt planeaba reducir el tipo de interés legal del 5\% al 4\%. Como dice Stark, jeso quería decir que más personas serían perseguidas por la usura!, pero, en la Introduction to the Principles of Morals and Legislation, Bentham no fue

45 Por tanto, se acerca a la figura del empresario descrita por Kirzner, en que el papel de la sorpresa y el descubrimiento son características de la función empresarial creativa [véase Kirzner (1998: 275-6) en Anexo; y Huerta de Soto (2000: 189)].

46 Como dice Dube, para Bentham el riesgo es un «placer» anticipado. Los placeres son más intensos cuanto más inciertos [Dube (1991: 97)], contra lo que hubiera afirmado Hume respecto a la incertidumbre.

47 Sin embargo, para considerar ese proceso de sorpresa no es necesario que haya utilidades subjetivas "previas al mercado» [para un análisis del proceso de sorpresa, véase Huerta de Soto (2001: 61)].

48 Stark (1952c: 425), extractado de The Psychology of Economic Man: VII.

49 Spengler (1975: 397-400). Una visión intermedia de la innovación es la que plantean las teorías evolucionistas del cambio económico, con transformaciones de la organización cumulativas y basadas en la «selección natural». Las empresas están motivadas por el beneficio pero sus acciones no son maximizadoras, aunque tienen en determinado momento ciertas capacidades y reglas de decisión [véase Nelson y Winter (1982)].

50 La semejanza de la teoría de Bentham con la de Hayek es obvia, como dice Dube [véase Dube (1990: 71-87)]. 
capaz de encontrar un lugar apropiado para la usura, con lo cual era una ofensa «imaginaria».

La Usura, que si debe ser una ofensa es una ofensa cometida con consentimiento, es decir, con el consentimiento de la parte supuestamente dañada, no puede merecer un lugar en el catálogo de las ofensas, a no ser que el consentimiento fuera obtenido injustamente o no libremente: en el primer caso, coincide con el fraude; en el segundo, con la extorsión. ${ }^{51}$

Además, entre los efectos perjudiciales de fijar un máximo de tipo de interés, Bentham enfatiza el decrecimiento en el número de posibles prestamistas y la formación de un mercado negro de crédito. Eso limitará las ganancias del banquero y éste será más cauteloso en sus fijaciones de los márgenes de riesgo implicados en los proyectos. Los préstamos se concederán sólo a los empresarios que operan en caminos de producción y distribución conocidos, con bajo riesgo. Así, Bentham considera que el efecto de una tasa de interés máxima será bloquear cualquier innovación y el mecanismo de desarrollo mismo. Por tanto, es necesario confiar en las fuerzas del mercado.

Además, en su Defence le reprochó a Smith que el mismo odio a la palabra «usura» y los prejuicios morales eran los responsables de la ley de la Usura. Aquí, Bentham erraba los argumentos de Smith, dado que éste no estaba hablando de una justicia moral, sino de que la tasa de mercado estaba de acuerdo con las realidades económicas dadas y sería el «precio por el tiempo» que aceptaría el hombre solvente (dado que, según Smith, el hombre es generalmente prudente). En cualquier caso, Bentham acabó su Defensa de la Usura en mayo de 1787 y mandó el manuscrito a George Wilson, un abogado de Lincoln`s Inn que lo dio a imprenta. El libro apareció hacia el fin de 1787. En una carta a Bentham el 4 diciembre de 1789, Wilson escribió:

51 Citado en Stark (1952a: 23), extractado de Introduction. 
¿Te dijimos alguna vez lo que el Dr Adam Smith dijo a Mr William Adam, el MP del Ayuntamiento, el verano pasado en Escocia? Las expresiones del Doctor fueron que «la Defensa de la Usura era el trabajo de un hombre superior, y que aunque le había dado algunos duros golpes, estaba escrito de una manera tan elegante que no podía quejarse, y pareció admitir que tú estabas en lo cierto». 52

Bentham se refirió a esta conversión supuesta en su segunda edición de Defence, en 1790, pero fue cuidadoso al decir que no conoció la conversión de Smith directamente. Buscó el reconocimiento abierto del cambio de opinión y mandó una carta a Smith, que éste recibió en los últimos días de su vida. Todo lo que hizo Smith fue mandar a Bentham una copia dedicada de La Riqueza de las Naciones, como reconoce Bentham en The True Alarm (escrito en 1801). Y es difícil de creer la conversión de Smith, que, de hecho, con el tiempo reforzó la importancia del hombre prudente en su sistema. Smith y Bentham tenían dos visiones del desarrollo económico: en Defence, Bentham lo considera caracterizado por cambios continuos determinados por mejoras, y, por tanto, con una tendencia no lineal; Smith considera que es lento, gradual, uniforme y no susceptible de variaciones repentinas.

La Defence en seguida fue reseñada y elogiada por el Monthly Review de mayo de $1788^{53}$. Además de apreciada en Inglaterra (la cita y defiende Thomas Reid), Defence fue muy apreciada en Francia (la cita y defiende Mirabeau) o en América, donde tuvo gran influencia (se adoptaron sus principios en el Estado de Mississippi en 1817, en Alabama, Virginia y New Hampshire). Los liberales aceptaron sus argumentos, y el proyecto de ley presentado el 15 de febrero de 1788 en Irlanda, apoyando una reducción del máximo legal al tipo de interés, fue denegado,

52 Rae (1895: 423 y ss).

53 Stark (1952a), extractado de Introduction. 
tal vez basándose en la Defence publicada en Dublín ese mismo año. Defence también recibió críticas, pero la primera de ellas aparece 30 años después de la fecha original del ataque de Bentham ${ }^{54}$.

En cualquier caso, como dice Stark, la literatura especializada admite que Defence of Usury debe su éxito más a la brillantez estilística que a la carga de profundidad de pensamiento ${ }^{55}$. Bentham se estaba haciendo famoso por defender la libertad de mercado, más allá que «el mismo Adam Smith»y, sin embargo, su argumento estaba siendo consciente «tramposo». Su argumento consistía en preguntarse por qué debemos dejar todos los demás precios fijos excepto el máximo de tipo de interés. Sin embargo, el mismo Bentham pidió que se estableciese un precio máximo sobre el grano en Defence of a Maximum — aunque esta obra no se llegó a publicar-y ahí se defiende de la inconsistencia reconociendo que, efectivamente, en Defence of Usury argumentaba contra un máximo - buscando polemizar con Smithy ahora, en Defence of a Maximum, como diría en el título, estaba a favor de un máximo - buscando polemizar con Long ${ }^{56}$.

54 Por ejemplo, la de Grahame, 1817, Defence of Usury Laws against the Arguments of Mr Bentham; la de Robert Maugham, 1824, Treatise on the Principles of the Usury Laws; with Disquisitions on the Arguments against them by Mr Bentham; el anónimo de 1825, Reasons against the Repeal of the Usury Laws; Francis Neale, 1826, Essay on Money Lending... and... Answer to the Objections of Mr Bentham», o John Whipple, 1855, Free Trade in Money, con el subtítulo Stringent Usury Laws, the best Defence of the People against Hard Times.

55 En Stark (1952a: 33) de Introduction.

56 En este panfleto, que elaboró a finales de 1801, el problema que abordó Bentham fue el de la escasez de provisiones en general y de grano para el pan en particular que se desarrolló en 1799 y que amenazaba con hambrunas y miseria masiva. Probablemente Bentham no hubiera decidido tomar parte en las discusiones si no hubiera encontrado un adversario valioso, pero lo encontró. Fue Charles Long, que publicó en 1800 un panfleto bajo el título A Temperate Discussion of the Causes which have led to the present High Price of Bread. Rechazaba la exigencia del pueblo que pedía la imposición por estatuto de un precio máximo del trigo. Bentham en principio no defendía esa medida pero, ante los argumentos de Long, pensó más en el tema y llegó a la conclusión de que una ley de máximo sería una medida defendible. Sin máximo, los cultivadores y comerciantes pueden querer retener 
De hecho, tras 14 años, Bentham vuelve al argumento del Defence of Usury y, aunque no se retracta, en el Prefacio reconoce que la fijación de un tipo de interés máximo tiene un mérito que no se le había ocurrido en 1797 y que podría haber aprobado la medida de máximo tipo de interés: hace posible que el gobierno tome prestado en términos más favorables, especialmente en tiempo de guerra. En última instancia, Bentham podría haber defendido la reducción del tipo de interés al modo keynesiano. He oído que en Inglaterra se propone reducir el tipo de interés para beneficiar al comercio... Eso no es lo que dice Smith, que sólo quería desincentivar a los pródigos y proyectistas ${ }^{57}$. Sin embargo, tal vez para seguir manteniendo la tesis que defendía al principio, en Institute afirma que la política de dinero barato con una reducción forzada de los tipos de interés supone un impuesto directo sobre los prestamistas que no incrementará, sino que reducirá, el capital, al llevarlo a países con mayor tipo de interés ${ }^{58}$.

Para los economistas modernos, el más notable defecto de la Defence of Usury es la ausencia en ella de una teoría del interés. Pero en Defence hay ideas que luego Bentham desarrolla en la teoría del interés y del capital. Se negaba a aceptar que el dinero fuera estéril, pero tampoco se veía atraído por una teoría de la productividad física. Se acerca más a una teoría monetaria del tipo de interés, en que la expectativa se hace básica. Así lo

\footnotetext{
lo que tienen del bien con la esperanza de un posterior incremento de los precios y beneficio. Y frente a la objeción de Long de que el consumo depende del precio y un precio bajo incentivará el consumo, Bentham toca el problema marshalliano de la elasticidad de la demanda, a pesar de que no lo elabora [Stark (1952c: 247302), extractado de Defence of a Maximum containing a particular examination of the arguments on that head in the pamphlet of 1800 attributed to a late Secretary to the Treasury to which are subjointed hints respecting the selection of radical remedies against dearth and Scarcity 1801].

57 Stark (1952a: 192), extractado de [Proposed] Preface [to the Second Edition] of Defence of Usury.

58 Para delimitar el carácter liberal de Bentham en lo que respecta al mercado del crédito, véase Leloup (2002: 7-20).
} 
demuestra su definición de préstamo como un intercambio de dinero presente por dinero futuro ${ }^{59}$, o la de ahorrador como aquel que ha realizado la decisión de sacrificar el presente por el futuro ${ }^{60}$. En carta III relata el deseo de un hombre de ahorrar, y su grado de ahorro, como la respuesta a

cuál de los dos puede considerarse un dolor más agudo y valorable, el dolor presente de controlar los deseos presentes, o el dolor contingente futuro a que puedo estar expuesto por la necesidad a la que puede reducirme en el futuro el gasto de gratificar estos deseos presentes ${ }^{61}$.

El mantenimiento de la ley de la usura, según Bentham, sólo puede venir de un prejuicio moral porque:

Aquellos que tienen la determinación de sacrificar el presente por el futuro son objetos naturales de envidia de aquellos que han sacrificado el futuro por el presente. Los niños que se han comido su tarta son los enemigos naturales de los niños que tienen la suya. ${ }^{62}$

\section{EL AHORRO FORZOSO}

Bentham considera que el dinero no es neutral. La fijeza de rentas nominales de ciertos grupos con alta propensión a consumir, como los rentistas, en un contexto de inflación lleva a que se produzca una transferencia de sus ingresos a los pro-

59 Stark (1952a: 132), extractado de Defence of Usury: Letter II.

60 Stark (1952a: 159), extractado de Defence of Usury: Letter X.

61 Stark (1952a: 133), extractado de Defence of Usury: Letter III. En otros ensayos se expresa de forma semejante, y el préstamo se ve «no como el riesgo de prestar sino como la mera auto-negación por no gastar» [Stark (1952 b: 294), extractado de Abstractor compressed view of a Tract intituled Circulating Annuities]. Hemos de decir que Bentham anticipa no sólo a Menger, Jevons o Marshall, sino que a la explicación de Boehm-Bawerk del tipo de interés, diciendo que si el valor del placer futuro depende no sólo de la intensidad y duración, sino de la certidumbre y cercanía, es más pequeño cuanto más incierto y más distante. Por tanto, surgirá un premio de riesgo.

62 Stark (1952a: 159), extractado de Defence of Usury. 
ductores con alta propensión a ahorrar, lo que provoca formación de capital (ahorro forzoso).

Por tanto, Bentham introduce una teoría del ahorro forzoso, o, como él la llama, la «Frugalidad forzosa», que luego desarrollará en el Institute en 1804 (casi al tiempo que Thornton en 1802), y que explica las conexiones y mecanismos de operación del dinero y se extenderá hasta la teoría de Keynes. Según Bentham el incremento de la cantidad de dinero favorece la acumulación de capital, aún a costa $-\mathrm{y}$ precisamente a costa- de la reducción del consumo de ciertos individuos con rentas fijas, que no reciben los beneficios de su ahorro. Por ejemplo, los precios se habían doblado desde 1760 a 1804 y el poder de compra del dinero se había reducido a la mitad. El objetivo de Bentham era prevenir el mal en el futuro y compensar el daño a las rentas fijas en el pasado. Recordando la teoría de las expectativas racionales, Bentham afirma que en el caso de que el incremento de billetes sea inesperado, no se producirá el incremento de precios inmediatamente y el incremento de la cantidad de dinero podrá afectar positivamente a la actividad y empleo.

Se producirá el mismo mal y en el mismo grado que con el dinero de oro y plata hasta la misma cantidad; pero la magnitud del mal estará en proporción a lo inesperado de la adición, no a la cantidad absoluta; $y$, en forma de dinero metálico, el influjo no es susceptible de un cambio tan brusco como en forma de papel. ${ }^{63}$

El gobierno «tiene» la posibilidad de incentivar el ahorro a través de un mecanismo artificial, la creación de dinero (un impuesto inflacionario...); sin embargo, no «debe» hacerlo porque es injusto obligar a la gente a sacrificar riqueza futura por presente ${ }^{64}$.

63 Stark (1952b: 208), extractado de Circulating Annuities.

64 Hayek (1932: 123-33). 
Mantener las cosas en la proporción en que están actualmente debe ser y, en general es el objetivo del legislador. Su gran propósito es conservar la masa total de expectativas en tanto sea posible...65

Concluye que el incremento de papel moneda debe restringirse y el de dinero efectivo mantenerse bajo control, o, por lo menos, no positivamente incentivado. Son las principales políticas monetarias por las que Bentham aboga y que desarrollará en The True Alarm, en mayo de 1801, intentando atacar el panfleto de Walter Boyd Letter... on the Influence of the Stoppage of Issues in Specie... on the prices of provisions. En el panfleto, Boyd mostraba opiniones opuestas a las de Bentham. Según Boyd, la sobre emisión del Banco de Inglaterra era la causa de la inflación del momento. Consideraba responsable de la escasez general de bienes al Banco de Inglaterra, la política de sus directores y, en particular, la suspensión de pagos en especie. Sin embargo, Bentham creía que el Banco de Inglaterra no era culpable y que el problema estribaba en la libertad de los bancos provinciales.

\section{CONCLUSIONES}

Bentham, como vemos, presenta una teoría original, que atisba conceptos marginalistas, e incluso austríacos. Existe una continuidad temática entre las teorías de Bentham y la de los economistas, que analizaban los mismos tipos de procesos, aunque sea desde concepciones pre-analíticas diversas. Para Bentham, la felicidad es una «idea», la expectativa del futuro placer y dolor que influye en la acción interesada y esperada. Su metodología es subjetivista y diferencia entre bienes de orden superior e inferior, de producción o de consumo, basán-

65 Stark (1952c: 198), extractado de The True Alarm. 
dose en la cercanía al uso como parámetro diferenciador. Las ideas de Bentham están continuamente rozando la de la importancia del principio marginal. En Axioms, el lector encuentra una exposición clara de la idea de «mínimo sensible» de riqueza, una forma de utilidad marginal. Pero, además, formula también la idea de utilidad marginal decreciente, lo que le permite defender por una parte una tendencia hacia la igualdad; por otra un conservadurismo económico que minimice la decepción en la masa total de expectativas. Además, Bentham con su método geométrico que muestra las consecuencias estáticas sobre el placer de una transferencia estaba pensando en una escala que nos recuerda los gráficos de utilidad que posteriormente realizarían los economistas. Casi llega a formular el principio de equimarginalidad en el intercambio, aunque no logra definirlo con nitidez.

Por otra parte, el autor defiende a los proyectistas y especuladores y reprocha a Adam Smith el haber infraestimado el papel de esos «hombres de talento». Para Bentham el proyectista capta la utilidad del bien final anticipadamente. En su Defence of Usury criticó a Smith por haber defendido el máximo de tipos de interés: el proyectista encontrará dificultad en hallar financiación y se bloqueará cualquier posibilidad de innovación. Aunque Smith leyó la carta que Bentham le envió sobre este tema, hay evidencias para suponer que no se convirtió a la posición de Bentham. Las distintas concepciones de empresario y del crecimiento hacían irreconciliables las dos teorías. Además, difieren en su teoría del tipo interés: la teoría de Bentham se acerca a una teoría monetaria en que la expectativa se hace básica. Así lo demuestra su definición de préstamo como un intercambio de dinero presente por dinero futuro, o la de ahorrador como aquel que ha realizado la decisión de sacrificar el presente por el futuro.

Además, Bentham fue un introductor de la economía de los costes de información, y la economía del tiempo y el espacio. 
Si por alguna razón se pudiera solventar el problema de la agencia del gobierno, Bentham no tendría ninguna justificación contra la intervención. Sin embargo, este problema no se puede resolver, por la propia definición anárquica de la información, de la que el hombre se apropia individualmente.

Por último, Bentham considera que el dinero no es neutral por la fijeza de rentas nominales de ciertos grupos con alta propensión a consumir, como los rentistas, que en un contexto de inflación lleva a que se produzca una transferencia de sus ingresos a los productores con alta propensión a ahorrar, lo que provoca formación de capital. Nos recuerda a la teoría de las expectativas racionales afirmando que en el caso de que el incremento de billetes sea inesperado, no se producirá el incremento de precios inmediatamente y el aumento de la cantidad de dinero podrá afectar positivamente a la actividad y empleo. Sin embargo, critica este ahorro forzoso porque lleva a transformaciones en la propiedad y a decepciones indeseables en la expectativa de posesión.

\section{BIBLIOGRAFÍA}

Bentham, Jeremy (1981), Tratados de legislación civil y penal, Madrid, Editora Nacional.

Bonner, John (1995), Economic Efficiency and Social Justice: The Development of Utilitarian Ideas in Economics from Bentham to Edgeworth, Aldershot, Edward Elgar Publishing.

Campos Boralevi, Lea (1984), Bentham and the oppressed, Berlin, Walter de Gruyter.

Collison Black, R.D. (1988), «Bentham and the Political Economists of the Nineteenth Century», The Bentham Newsletter, 12, junio.

Dube, Allison (1990), «Hayek on Bentham», Utilitas, 2, 1, mayo, pp. 71-87. 
- (1991), The Theme of Acquisitiveness in Bentham's Political Thought, New York, Garland publising.

Halévy, Elie (1928), The Growth of Philosophic Radicalism, London, Faber and Faber.

HAYEK, Friedrich A.. (1932), «A note on the Development of the Doctrine of Forced Saving», The Quarterly Journal of Economics, vol. XLVI, Cambridge, Harvard University Press, pp. 123-133.

HÉBert, R.F. y Link, A.N. (1988), The Entrepreneur. Mainstream Views and Radical Critics, New York, Praeger.

Heberton Evans Jr, G. (1949), «The Entrepreneur and Economic Theory, a Historical and Analytical Approach», The American Economic Review, Papers and Proceedings, XXXIX, mayo, 337-38.

Hollander, S. (1973), The Economics of Adam Smith, London, Heinemann Educational Books.

Huerta de Soto, Jesús (2000), La Escuela Austriaca, mercado y creatividad empresarial, Madrid, Editorial Síntesis.

- (2001), Socialismo, cálculo económico y función empresarial, Madrid, Unión Editorial, 3. a edición [2005].

KIRZNER, Israel M. (1973), Competencia y empresarialidad, 2. ${ }^{a}$ edición, Madrid, Unión Editorial [1998].

KNIGHT, Frank H. (1921), Riesgo, Incertidumbre y Beneficio, Madrid, Aguilar [1947].

Leloup, Sandrine (2002), «El liberalismo económico, a prueba en Bentham: Panóptico y mercado de crédito», Télos. Revista Iberoamericana de Estudios Utilitaristas, X, 2, 2001, Santiago de Compostela, SIEU, pp. 7-20.

Menger, Carl (1984), Investigations into the Method of the Social Sciences with Special Reference to Economics, New York, New York University Press [1883].

Mises, Ludwig von (1949), La Acción Humana. Tratado de Economia, 7. a edición, Unión Editorial, Madrid [2004]. 
Nelson, Richard R. y Sydney, Winter G. (1982), An Evolutionary Theory of Economic Change, Cambridge, The Belknap Press of Harvard University Press.

Pesciarelli, Enzo (1989), «Smith, Bentham and the Development of Contrasting Ideas on Entrepreneurship», History of Political Economy, 21, 3, 521-36.

Popper, Karl (1957), La miseria del historicismo, Madrid, Taurus [1961].

RAE, John (1895), Life of Adam Smith, Londres, Macmillan.

Rodríguez Braun, Carlos (1989), La cuestión colonial y la economía clásica, Madrid, Alianza.

Rothbard, Murray N. (1982), La ética de la libertad, Madrid, Unión Editorial [1995].

Santos Redondo, Manuel (1997), Los economistas y la empresa. Empresa y empresario en la historia del pensamiento económico, Madrid, Alianza Editorial.

Schumpeter, Joseph A. (1949), Change and the Entrepreneur, Research Center in Entrepreneurial History, Harvard University.

- (1954), History of Economic Analysis, Oxford, Oxford University Press.

- (1949), «La teoría económica y la historia empresarial», Ensayos, Barcelona, Oikos-Tau [1966], pp. 255-272.

SchwArTz, Pedro (1976), «La influencia de Bentham en España», Información Comercial Española, n. ${ }^{\circ}$ 517, septiembre, pp. 52-56.

- (1986), «Jeremy Bentham’s Democratic Despotism», Ideas in Economics, ed. by R.D. Collison Black, Basingstoke, Macmillan Press, pp. 74-103.

Spengler, Joseph J. (1975), "Adam Smith and Society's Decision-Makers», Essays on Adam Smith, Skinner. A.S. y Wilson, T. (eds.), Oxford, pp. 397-400.

StARK, Werner (1952a), Jeremy Bentham's Economic Writings. Critical Edition Based in his Printed Works and Unprinted Manu- 
scripts, vol. 1, Londres, Published for The Royal Economic Society by George Allen \& Unwin Ltd.

- (1952b), Jeremy Bentham's Economic Writings. Critical Edition Based in his Printed Works and Unprinted Manuscripts, vol. 2, Londres, Published for The Royal Economic Society by George Allen \& Unwin Ltd.

- (1954), Jeremy Bentham's Economic Writings. Critical Edition Based in his Printed Works and Unprinted Manuscripts, vol. 3, Londres, Published for The Royal Economic Society by George Allen \& Unwin Ltd. 